\title{
Effects of Gaze Shifts on Maintenance of Spatial Memory in Macaque Frontal Eye Field
}

\author{
Puiu F. Balan and Vincent P. Ferrera \\ Department of Psychiatry, Center for Neurobiology and Behavior, and David Mahoney Center for Brain and Behavior Research, Columbia University, New \\ York, New York 10032
}

The activity of 91 neurons in the frontal eye fields (FEFs) of two macaque monkeys was recorded while the animals performed a delayed spatial match-to-sample task. During the delay, the animals were required to shift their gaze to one of four eccentric locations. Neuronal activity during the delay was analyzed for sensitivity to cue location and eye position. One-third of the neurons showed significant delay activity selective for cue location, whereas slightly more than one-half of the neurons showed significant modulation of delay activity when the gaze was shifted to an eccentric location. Despite this modulation, the neurons continued to signal their preferred cue location during most of the delay. However, after recentering saccades, the memory signal was temporarily abolished and then reemerged over a period of few hundred milliseconds. This is consistent with the idea that spatial working memory is buffered outside of the FEF. For most neurons, delay activity tended to increase when the gaze was shifted away from the preferred location and to decrease when the gaze was shifted toward the preferred location. This pattern of modulation is consistent with a vector subtraction mechanism that allows for the superposition of multiple saccade plans.

Key words: eye position; gain modulation; spatial memory; saccade planning; sensorimotor transformation; prefrontal cortex

\section{Introduction}

The frontal eye field (FEF) contains neurons that exhibit spatially selective sustained activity as well as a variety of signals related to eye movements. Saccades can be evoked by electrical microstimulation of the FEF at low current amplitudes (Bruce et al., 1985). The FEF is involved in transforming visual signals into instructions for voluntary eye movements (Mohler et al., 1973; Bruce and Goldberg, 1985; Schall et al., 1995b; Ferraina et al., 2000). The visual activity of neurons in the FEF shows a predictive component during double-step (Goldberg and Bruce, 1990; Umeno and Goldberg, 1997) and triple-step (Tian et al., 2000) saccade tasks. FEF neurons are also activated when the remembered location of a flashed visual stimulus is brought into the receptive field (RF) by an eye movement (Tian et al., 2000; Umeno and Goldberg, 2001). These observations suggest that FEF receptive fields are remapped to compensate for the change in eye position caused by a saccade. This compensation is consistent with the effects of microstimulation in the FEF when the stimulus is applied at the onset of a voluntary saccade (Mushiake et al., 1999) or when two FEF sites are stimulated asynchronously (Fujii et al., 1998). However, when the gaze is held steady, the direction and magnitude of saccades evoked by FEF microstimulation show very little dependence on the initial orbital position of the

Received March 14, 2003; revised April 16, 2003; accepted April 17, 2003.

This work was supported by the EJLB Foundation and MH59244. We thank G. Case for designing and fabricating the multielectrodes used in this study and A. Barborica for designing the multichannel discriminator. Helpful discussion was provided by J. Bisley, S. Krishna, M. Goldberg, J. Gottlieb, J. Grinband, and D. Salzman. J. Willi and A. Banta provided excellent technical assistance.

Correspondence should be addressed to Dr. Vincent P. Ferrera, Center for Neurobiology and Behavior, Columbia University, 1051 Riverside Drive, Kolb Annex 504, New York, NY 10032. E-mail: vpf3@columbia.edu.

Copyright $\odot 2003$ Society for Neuroscience $\quad$ 0270-6474/03/235446-09\$15.00/0 eye (Russo and Bruce, 1993; Fujii et al., 1998). One might therefore predict that changes in eye position would have little effect on sustained activity in the FEF.

The FEF receives afferent input from parietal areas involved in spatial attention and movement planning (Barbas and Mesulam, 1981; Schall et al., 1995a). Eye position-dependent modulation of visual- and movement-related neuronal activity has been observed previously in many parietal areas (Andersen and Mountcastle, 1983; Andersen et al., 1985, 1990; Galletti et al., 1995; Bremmer et al., 1998), as well as the premotor cortex (Graziano et al., 1997; Mushiake et al., 1997; Graziano and Gross, 1998), including the supplementary eye field (Schlag et al., 1992). Eye position-dependent gain modulation is thought to be important in transforming retinotopically coded visual information into head-centered movement commands (Zipser and Andersen, 1988; Siegel, 1998; Salinas and Thier, 2000). The posterior parietal, premotor, and prefrontal cortex share many anatomical connections and neuronal response properties. From this standpoint, the presence of eye position effects in the FEF might not be surprising.

We examined the effects of eye movements on sustained activity by training monkeys to perform a delayed spatial match-tosample task with a simple motor perturbation; during the delay, monkeys were required to shift their gaze to one of four eccentric locations, hold that position for slightly longer than $1 \mathrm{sec}$, and then shift their gaze back to center. Approximately one-half of the FEF neurons we recorded showed activity modulation when the gaze was shifted during the delay. In spite of this, delay activity tended to signal the preferred retinal location regardless of eye position. However, after the gaze was recentered, tuning for cue location disappeared, as if the memory for cue location were 
erased. The tuning then reemerged over a period of a few hundred milliseconds to signal the remembered cue location. These observations suggest that spatial working memory is buffered outside of the FEF, which then reads out the contents of the memory buffer during saccade planning. In addition, delay activity showed a tendency to increase when the gaze was shifted away from the receptive field and to decrease when the gaze was shifted toward the receptive field. This is consistent with a vector subtraction mechanism (Quaia et al., 1998) that could serve to remap saccade goals based on the recent history of eye movements or to represent a superposition of multiple saccade plans.

\section{Materials and Methods}

Experiments were performed on two juvenile male rhesus monkeys ( $\mathrm{Ma}$ caca mulatta). All of the methods were approved by the Institutional Animal Care and Use Committee at Columbia University and the New York State Psychiatric Institute. Monkeys were prepared for experiments by surgical implantation of a post used for head restraint and a recording chamber to give access to the cortex. A monocular scleral search coil was implanted for eye position recording (Judge et al., 1980).

Visual stimulation. Visual targets were generated and controlled by a Cambridge Research Systems (Cambridge, UK) VSG2/3F video frame buffer with an on-board microprocessor. The output from the video board was displayed on a calibrated color monitor (Mitsubishi, Tokyo, Japan) with a $60 \mathrm{~Hz}$ noninterlaced refresh rate. The spatial resolution of the display was 1280 pixels by 1024 lines. The frame buffer was programmed to send out digital pulses (frame sync) for timing purposes at the beginning of each video frame in which a target was turned on or off. These pulses were recorded by the computer using a hardware timer and stored together with the eye movement data.

Fixation and saccade targets were small $\left(0.5\right.$ and $1.0^{\circ}$, respectively) yellow squares of $15.0 \mathrm{~cd} / \mathrm{m}^{2}$ luminance presented on a uniform dark background. Except for the targets, the subject was in total darkness during each trial. Between trials, there was dim background illumination. The background luminance of the cathode ray tube monitor was below the threshold of our photometer $\left(0.2 \mathrm{~cd} / \mathrm{m}^{2}\right.$; OptiCal; Cambridge Research Systems) and below the detection threshold of dark-adapted human observers.

Behavioral task. We trained monkeys to perform a delayed spatial match-to-sample task (see Fig. 1). At the start of each trial, monkeys fixated a target in the center of the screen. Then a peripheral cue was flashed for $100 \mathrm{msec}$ at one of four locations (up, down, right, or left). The cue eccentricity was adjusted (within screen limits) for each recording site. The mean eccentricity over all of the sites was $8.8^{\circ}$ (range, $8-14^{\circ}$ ). The monkey maintained fixation for an additional $2250 \mathrm{msec}$ memorydelay interval. At the end of the delay, the fixation target disappeared, and two identical choice targets appeared simultaneously. One target was at the location of the cue, and the other was at an equal eccentricity but in the opposite direction relative to fixation. The monkey was rewarded for making a saccade to the target that matched the cue location. On onefifth of the trials, the fixation position remained at the center of the screen for the entire delay. For the other trials, the fixation target stepped to one of four locations: up, down, right, or left of the initial fixation position. The step size was equal to the cue eccentricity. The step occurred 250 msec after the start of the delay interval. The fixation target remained at the eccentric location for $1500 \mathrm{msec}$ and then stepped back to the center of the screen for the last $500 \mathrm{msec}$ of the delay. Each time the fixation target moved, the monkey was required to shift his gaze to its location. A complete block of trials comprised four cue directions and five eye positions, randomly interleaved, for a total of 20 trials.

Neuronal recording and stimulation. Stainless-steel or plastic recording chambers were implanted at stereotaxic coordinates of 15-18 lateral and $20-25$ anterior, in accordance with studies of the frontal eye field described previously (Robinson and Fuchs, 1969). Neuronal activity was recorded using platinum-tungsten 8-trode microelectrodes (typical impedance, $0.5 \mathrm{M} \Omega$ ). The electrode signal was amplified, filtered, and passed through a time-amplitude window discriminator to separate action potentials from background noise. Amplification, filtering, and dis-

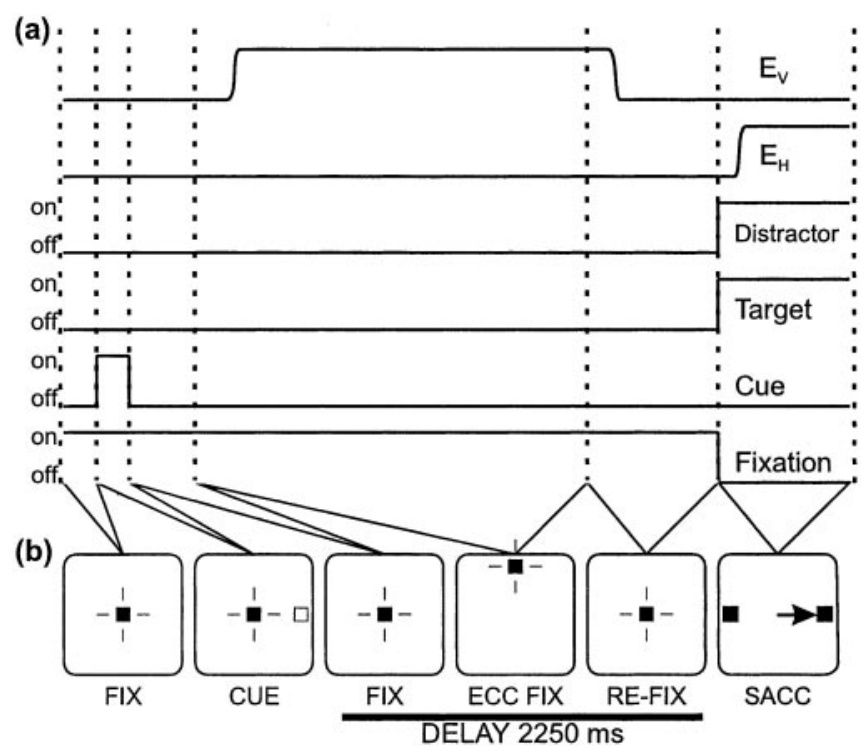

Figure 1. Delayed spatial match-to-sample task. $a$, Timing of trial events: fixation (FIX) (100 $\mathrm{msec})$, cue presentation (CUE) (100 msec), delay [fixation (FIX) (250 msec), eccentric/central fixation (ECC FIX) (1500 msec), and refixation (RE-FIX) (500 msec)], and choice saccade (SACC) (500 msec). b, Schematic of visual display during task. Black squares indicate fixation target (thin lines are for illustration only; they were not visible on display) or choice targets. White square indicates cue. Arrow indicates choice saccade. Target and Distractor are the two identical choice stimuli that appeared simultaneously near the end of the trial. On/off indicate the visibility of the stimulus. $E_{H}$ and $E_{V}$ are horizontal and vertical eye position, respectively. The horizontal black bar groups the panels that belong to the delay period.

crimination were performed by a digital signal processing-based multichannel slope/height window discriminator (MCD) designed in our lab. Using the multielectrode and MCD, we were able to record up to six neurons simultaneously. The time of each action potential was recorded with a resolution of $0.02 \mathrm{msec}$.

Electrical microstimulation was used to determine whether recording sites were located within the functionally defined FEF (Bruce et al., 1985). Sixty-seven millisecond trains of biphasic pulses $(0.2 \mathrm{msec} / \mathrm{phase} ; 350$ $\mathrm{Hz}$ ) were delivered while monkeys fixated a central target, which was turned off for $200 \mathrm{msec}$ before the electrical stimulus was delivered (Opris et al., 2001). Pulse amplitude was varied between 0 and $100 \mu \mathrm{A}$ to ascertain the threshold for electrically evoked saccades. Recording sites were assigned to the FEF if the stimulation threshold was $\leq 50 \mu \mathrm{A}$ (Bruce et al., 1985).

Eye movement recording and analysis. Eye position was monitored using a scleral search coil system (CNC Engineering, Seattle, WA). Separate horizontal and vertical eye position signals were fed through an analog differentiator (low pass; $-3 \mathrm{~dB}$ at $25 \mathrm{~Hz}$ ) to yield horizontal and vertical eye velocity. The eye position and eye velocity signals were then digitally sampled by computer at $500 \mathrm{~Hz}$ per channel and stored on disk for off-line analysis. Eye position and velocity records were used to estimate saccade latency and amplitude. Saccade onsets and end points were computed using an acceleration criterion.

Data analysis. Neural and behavioral data were analyzed in Matlab (MathWorks, Natick, MA). Firing rates (FRs) were subjected to two-way ANOVA (cue direction and eye position; $p<0.05$ ). Preferred cue vectors (PCVs) were constructed by calculating the weighted average (i.e., center of mass) of the delay activity for different cue directions when the eye position was in the center of the screen. Preferred eye vectors (PEVs) were constructed by first averaging delay activity over cue direction for each eccentric eye position. Then the weighted average of activity for the four eccentric eye positions was computed. Circular statistics (Rayleigh's uniformity test; modified Rayleigh or $V$ test; Hodges-Ajne test) and multivariate ANOVA (Hotelling-Lawley trace test) were derived from Zar (1999). Statistical tests and other characterizations of the data will be described in more detail as they are introduced in Results. 


\section{Results}

We recorded from 91 neurons in the anterior bank of the arcuate sulcus in two monkeys. Of these, 70 were located at lowthreshold $(\leq 50 \mu \mathrm{A})$ stimulation sites, and the remaining 21 were within $1 \mathrm{~mm}$ of a low-threshold site. We analyzed neuronal firing rates during four intervals of the task: (1) the cue (100 msec while the peripheral cue was illuminated), (2) early delay after the cue and before the first saccade, (3) the middle part of the delay (750-1750 msec after the start of the delay period), and (4) late delay after the recentering saccade and before the choice saccade (Fig. 1). To test the reliability of cue direction and eye position effects on activity in the middle delay, we performed a two-way ANOVA on each cell. This analysis resulted in 30 of $91(33 \%)$ cells with a significant effect $(p<0.05)$ of cue direction, 47 of $91(52 \%)$ with a significant effect of eye position, and 16 of 91 (18\%) with a significant interaction. The number of cells that showed a significant effect for both cue direction and eye position was 25 of $91(27 \%)$, which is somewhat greater than the $18 \%$ that would be expected if these two properties were independently distributed across the population.

How does activity modulation related to gaze shifts interact with the memory of cue location? Figure 2 shows the activity of a single neuron for trials in which the remembered cue was presented inside the RF. Three different gaze positions are shown in Figure 2: gaze shifted away from the RF $(a)$, gaze held at center position $(b)$, and gaze shifted toward the RF $(c)$. Shifting gaze clearly modulated the delay activity, but how did this affect the tuning of the neuron? Figure 2, middle row, shows mean firing rate during the middle delay as

a function of cue direction for each of the three eye positions. For this neuron, it appeared that shifting gaze away from the RF enhanced the tuning (as indicated by the length of the weighted vector sum) (Fig. 2d), while shifting toward the RF abolished tuning $(f)$. Figure 2, bottom row, shows tuning curves during the late-delay interval. When eye position returned to center after eccentric gaze shifts (Fig. $2 g, i$ ), tuning was poor regardless of whether the gaze shift had been toward or away from the RF.

The tuning of each neuron was summarized by calculating the weighted vector sum for each eye position as follows:

$$
V(e)=\sum_{\theta} F R(e, \theta) \cdot \boldsymbol{u}(\theta)
$$

where $V(e)$ is the tuning vector for each eye position, $\operatorname{FR}(e, \theta)$ is the firing rate for each eye $(e)$ and cue $(\theta)$ direction, and $\boldsymbol{u}$ is a unit vector with direction $\theta$. To examine tuning dynamics at the population level, we constructed population vectors by summing $V(e)$ over all of the neurons. Before summing, $V(e)$ for each neuron was rotated according to the preferred direction of the cell. b

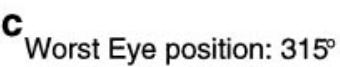
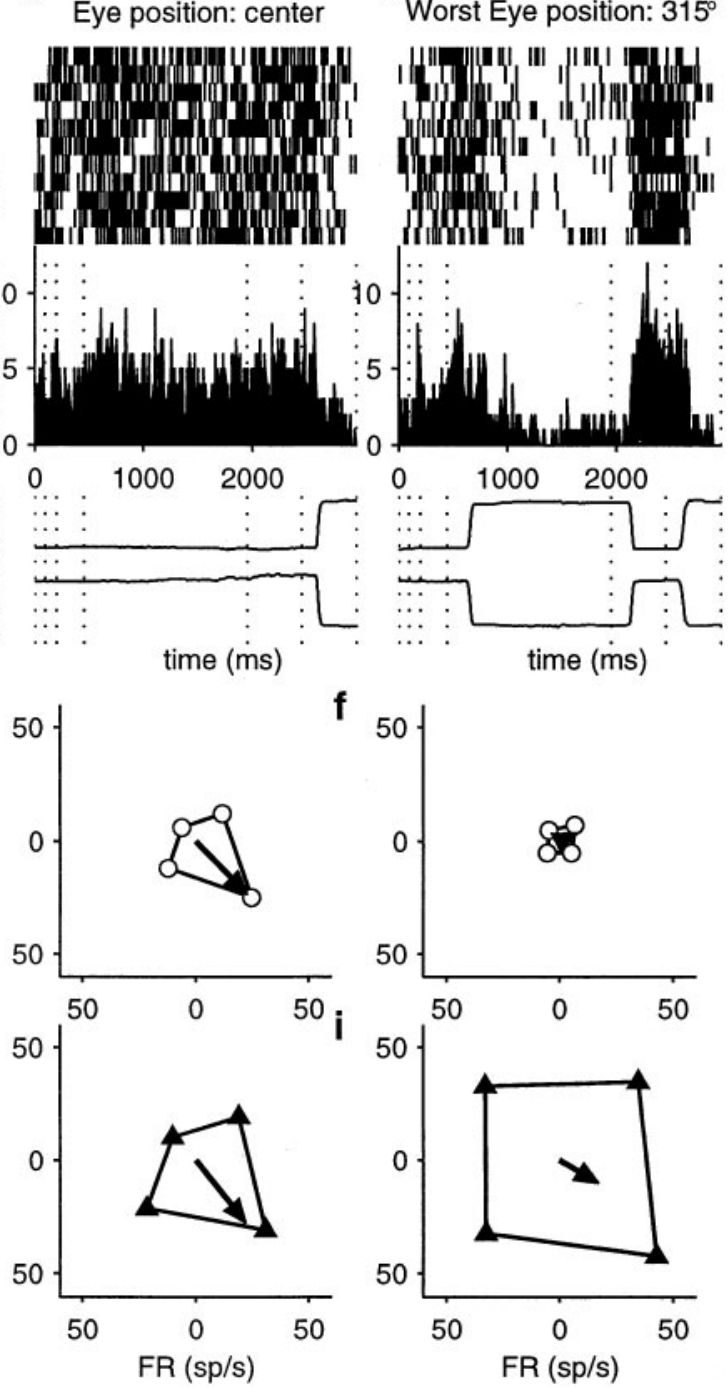

Figure 2. Example of middle-delay and late-delay interval activity and tuning dynamics. $a-c$, Spike rasters and histograms (smoothed with a Gaussian; width, $12 \mathrm{msec}$ ). $\mathrm{E}_{\mathrm{H}}$ and $\mathrm{E}_{\mathrm{V}}$ are horizontal and vertical eye position, respectively, for a single representative trial. $d-f$, Responses as a function of cue direction and tuning vectors for middle-delay activity. $g-i$, Spatial tuning for late-delay activity. sp/s, Spikes per second.

The preferred direction was taken to be the $V(e)$ direction computed from delay activity when the gaze was at the center position. Population vectors were computed for a succession of overlapping time intervals (interval width, $200 \mathrm{msec}$; center-to-center spacing, $100 \mathrm{msec}$ ). For this analysis, we used activity during the early delay (after the end of the cue presentation and before the first saccade) and middle delay (1000 msec period starting immediately after the first saccade). For center fixation trials, the corresponding time intervals were determined on the basis of the average latency of the first and second saccades. The second time interval was $\sim 200-250 \mathrm{msec}$ earlier than the 750-1750 msec interval used in the previous analysis to assess tonic delay activity. Figure 3 shows population vector dynamics during the two portions of the delay interval. Although there was considerable variability in the tuning vectors for individual neurons, the population vectors were within $\pm 45^{\circ}$ (often within $\pm 30^{\circ}$ ) of the preferred cue direction. For some gaze positions (Fig. 3e), there appeared to be a systematic bias, but this bias was also present during the first part of the delay, when the gaze was at the center position. Hence, the bias 


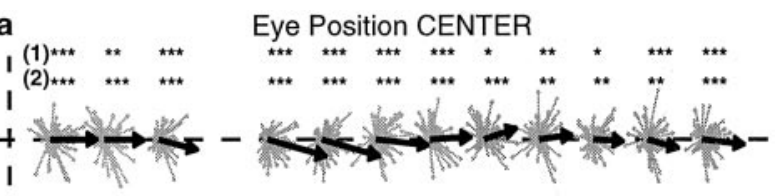

I

b Eye Position relative to Preferred Cue Direction is $0^{\circ}$

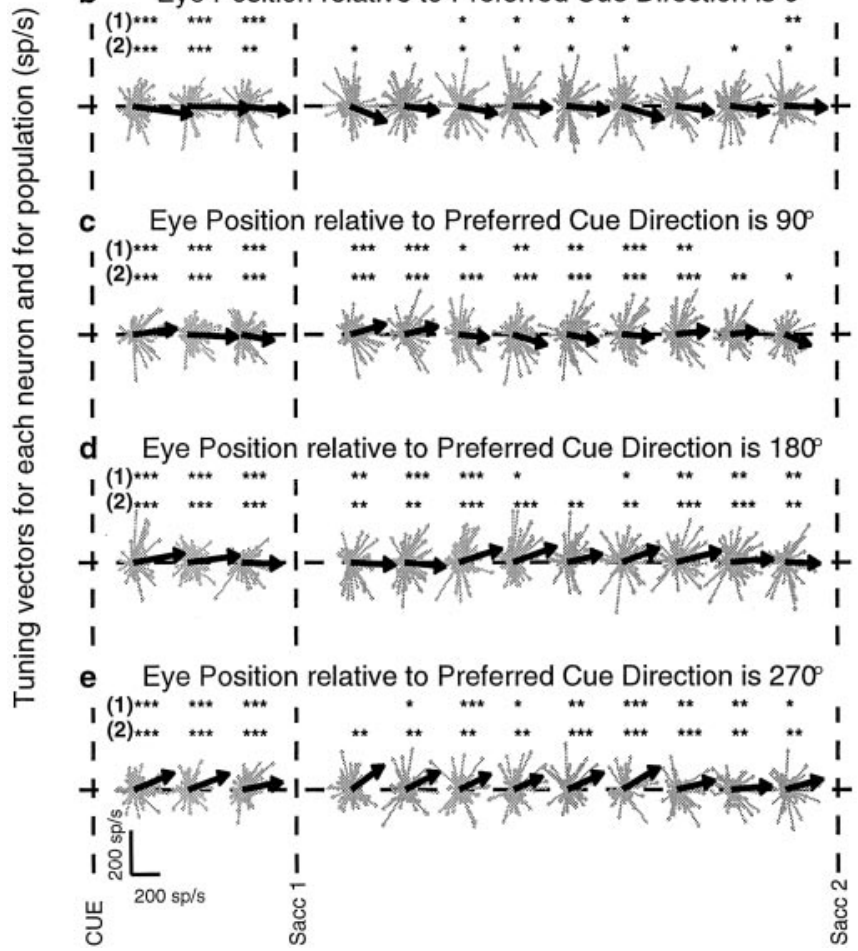

Figure 3. Population vector dynamics for early-delay and middle-delay activity. Each row represents a different eye position during the delay. Small arrows are tuning vectors (magnitude, $20 \times$ ) for individual neurons. Large arrows are population vectors. Horizontal dashed lines indicate preferred cue direction for center eye position during delay. Vertical dashed lines indicate the early and middle delay. Asterisks indicate significance level of Rayleigh uniformity test for neuronal tuning vector directions (1) and Hotelling-Lawley test for population vector amplitude (2). sp/s, Spikes per second; Sacc, saccade.

was probably not induced by the gaze shift but was an artifact of limited sampling (in terms of the number of neurons as well as the number of directions). The Rayleigh and Hotelling-Lawley tests were used to test the statistical significance of the direction and amplitude, respectively, of each population vector (Fig. 3). Overall, a memory of the cue location appears to be preserved across the initial shift in gaze.

We next looked at what happened when gaze shifted back to center and the final choice saccade was made. Figure 4 shows the tuning vectors for individual neurons and population vectors based on activity for the entire delay (leftmost column) and the period between the recentering and choice saccades (note that the scale is approximately twice that of Fig. 3). When the eye remained at center fixation (Fig. $4 a$ ), there was only a slight increase in the population vector magnitude as the time of the choice saccade approached. However, when there was a shift of gaze from an eccentric location back to the center, the population vector vanished in all of the cases (Fig. $4 b-e$ ) for the first $100 \mathrm{msec}$ after the saccade. The population vector then reemerged and continued to increase in magnitude as time for the final saccade approached. The disappearance of the population vector after the recentering saccade was not attributable to cancellation of the tuning vectors for individual neurons with opposite direction, a

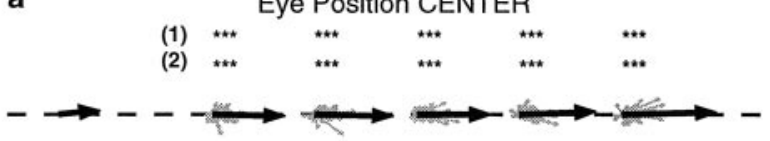

b Eye Position relative to Preferred Cue Direction is $0^{\circ}$

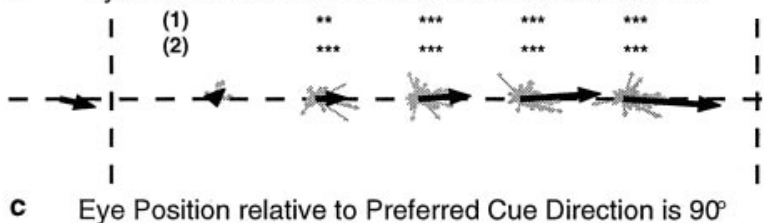

C Eye Position relative to Preferred Cue Direction is $90^{\circ}$

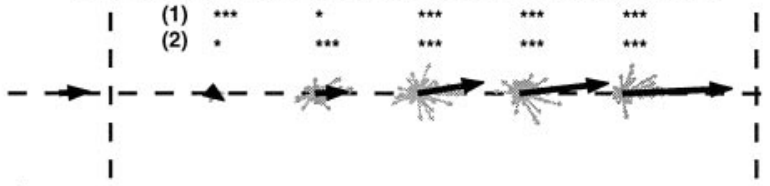

d Eye Position relative to Preferred Cue Direction is $180^{\circ}$

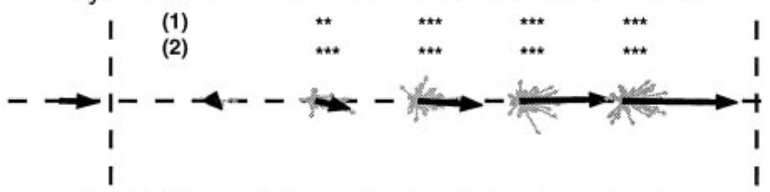

e Eye Position relative to Preferred Cue Direction is $270^{\circ}$

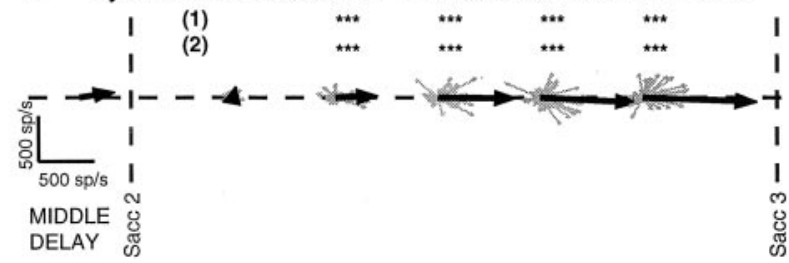

Figure 4. Population vector for middle-delay and late-delay activity. Each row represents a different eye position during the delay. Small arrows are tuning vectors (magnitude, $20 \times$ ) for individual neurons. Large arrows are population vectors. The leftmost arrow in each row indicates middle-delay population vectors. Horizontal dashed lines indicate preferred cue direction for center eye position during delay. Vertical dashed lines indicate the middle and late delay. Asterisks indicate significance level of Rayleigh test (1) and Hotelling-Lawley test (2). sp/s, Spikes per second; Sacc, saccade.

but to a general loss of tuning for all of the neurons. The Rayleigh test was used to establish that the distribution of individual tuning vector directions was nonuniform, and the Hotelling-Lawley test was used to establish the significance of the population vector amplitude (Fig. 4).

Changes in tuning over the time course of the entire delay are summarized plotting the magnitude of the population vectors (Fig. 5). For eccentric gaze positions (Fig. $5 b-e$ ), there is some suggestion that just after the first saccade, the population tuning is somewhat weaker compared with trials in which no saccade is made during the delay $(a)$, if only because the latter shows slight enhancement at the beginning of the delay. For the delay interval as a whole, tuning is slightly weaker for eccentric gaze positions than for the center gaze. It is after the second, recentering saccade that the tuning is temporarily lost.

So far, we showed that gaze shifts modulate delay activity in FEF, but we did not characterize the nature of the modulation. We next address the issue of how delay activity modulation varies with the direction of the gaze shift relative to the preferred direction of the neuron. A fundamental limitation of our experimental design is the following: because gaze shifts to eccentric locations were always followed by a sequence of two saccades, we cannot resolve whether the modulation was attributable to an eye posi- 


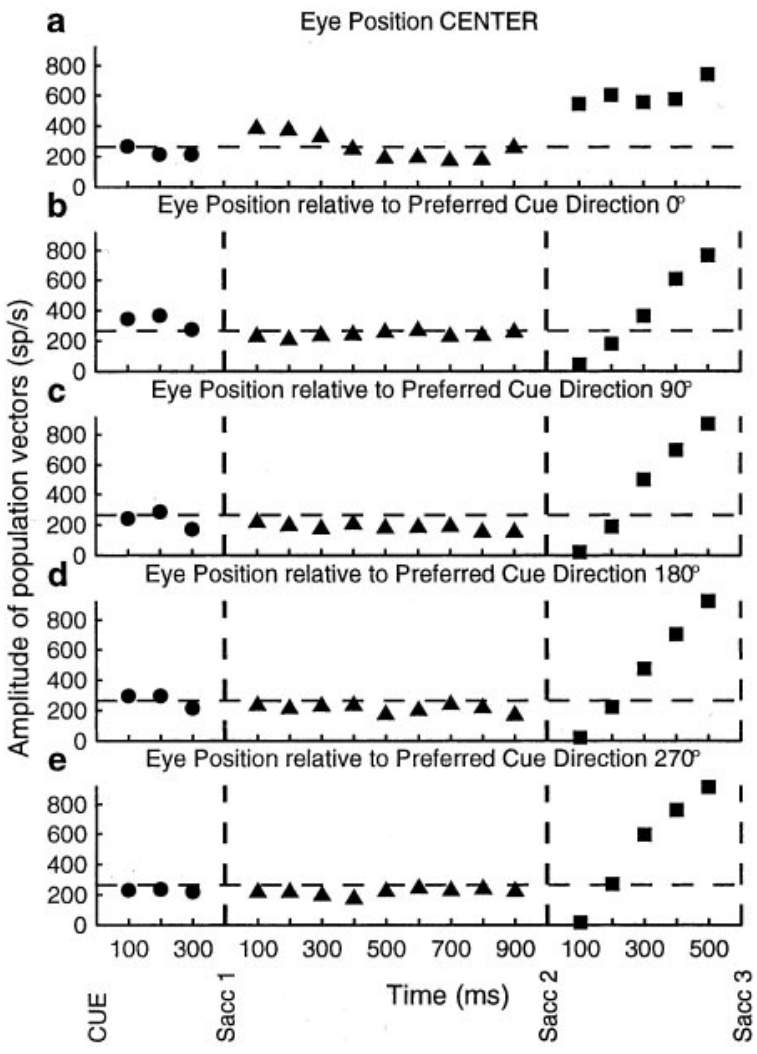

Figure 5. Population vector amplitude for entire delay. Each row represents a different eye position during the delay. Horizontal dashed lines indicate average delay activity for trials in which fixation remained at center. Vertical dashed lines separate the early, middle, and late delay periods. The filled circles, triangles, and squares represent the population vector amplitude at different moments in time during the early, middle, and late delay, respectively. sp/s, Spikes per second; Sacc, saccade.

tion signal or to the planning of multiple saccades. We will address this ambiguity below. For now, we will simply refer to the modulation as if it were an eye position signal.

Figure 6 shows data from two neurons that were representative of our sample. (Note that, for this and all of the subsequent analysis, we are dealing with tonic activity in the interval of 750$1750 \mathrm{msec}$ after the start of the delay.) The first neuron (Fig. 6a) was typical in that the gaze-dependent modulation was directionally tuned, and the best eye position was in a direction opposite the best cue direction. A second type of modulation had the form of a general enhancement (Fig. 6b) or suppression of activity for all of the eccentric gaze positions. This pattern was shown by only a few neurons.

We refer to the tuning vector for center fixation as the PCV, and this is computed for each neuron. A corresponding PEV was computed by first averaging activity for all four cue locations at each eccentric eye position. Then the weighted vector average of the mean delay activity for the four eccentric eye positions was calculated. We looked for a systematic relationship between these two vectors by comparing their directions. Figure $7 a$ shows the PEVs for each cell. Each PEV was rotated by subtracting the direction of the PCV for that cell. The directions of the PEVs were significantly nonuniform (Rayleigh test, $p<0.001$ ), with mean direction significantly different from $0^{\circ}(V$ test; $p=1.0)$ but not from $180^{\circ}$ ( $V$ test $\left.p<0.0001\right)$. The direction of the vector average of the rotated PEVs was $179.25^{\circ}$ (in this reference frame, the PCV directions were all equal to zero), and its amplitude was $2.41 \pm$ 0.63 (SEM) spikes/sec, which was significantly different from
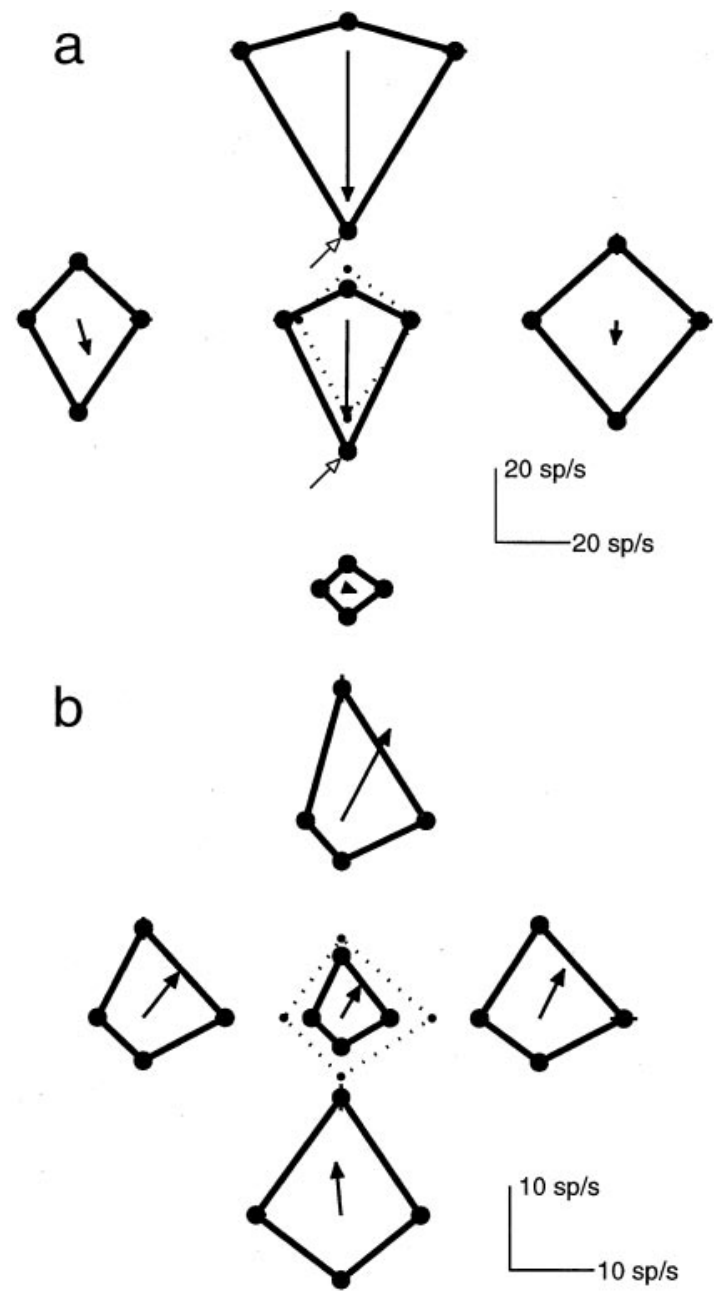

Figure 6. Two examples of neurons with cue and eye position modulation during the delay. $a$, Cell with preferred eye position opposite preferred cue location. Thick black lines represent delay activity as a function of cue location (error bars are \pm 1 SEM). Each tuning curve is offset to reflect the eye position during the delay. Filled arrows are the center-of-mass vector for each tuning curve. Open arrows indicate an example of trials with matching saccade. Dotted lines indicate the tuning curves for activity during the 100 msec cue interval. $b$, Cell with enhanced response for all of the eccentric eye positions. The conventions are the same as those in $a . \mathrm{sp} / \mathrm{s}$, Spikes per second.

zero (Hotelling-Lawley; $p<0.001$ ). The distribution of absolute differences between the PCV and PEV directions is shown in Figure $7 b$. This distribution is significantly nonuniform (Rayleigh test; $p<0.0001)$. There was a clear tendency for the preferred eye direction to be opposite to the preferred cue direction $(V$ test; $p<0.0001$ ).

The magnitude of the preferred eye and preferred cue vectors is an index of neuronal sensitivity to eye and cue position, respectively. Over the entire population of neurons, the mean PEV magnitude was $4.02 \pm 0.52$ (SEM) spikes/sec (median, 2.07). The mean PCV magnitude was $4.17 \pm 0.54$ (SEM) (median, 2.29). The PEV and PCV magnitudes were not significantly different (paired $t$ test; $p=0.673$ ). By adjusting for the cue and eye eccentricity, the preferred vector magnitude could be converted to a sensitivity of $\sim 0.24 \pm 0.03 \mathrm{spikes} \cdot \mathrm{sec}^{-1} \cdot \mathrm{deg}^{-1}$ for cue position and $0.23 \pm 0.03 \mathrm{spikes} \cdot \mathrm{sec}^{-1} \cdot \mathrm{deg}^{-1}$ for eye position.

Having shown that the cue and eye vectors are, on average, equal in magnitude but opposite in direction, we now examine more closely the issue of eye position versus saccade planning. 

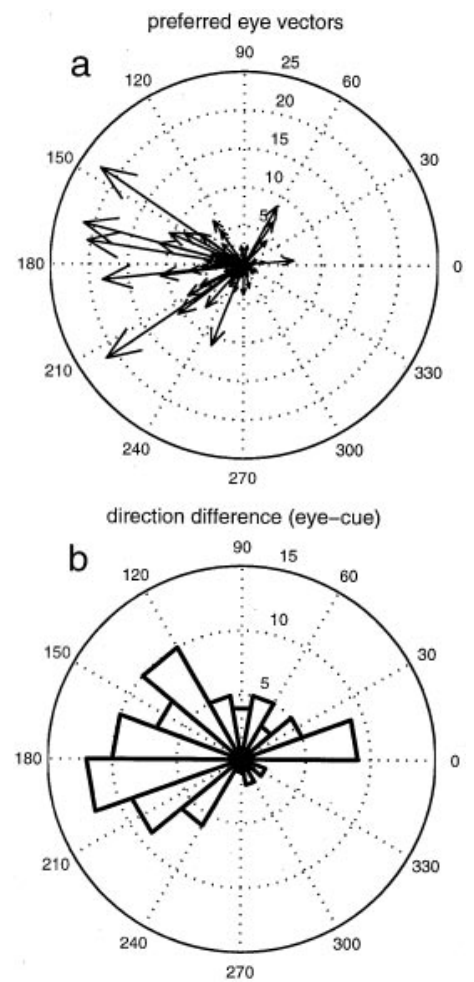

Figure 7. Comparison of best cue direction versus best eye direction. $a$, Arrows are preferred eye vectors for individual neurons. The PEVs have been rotated by subtracting the direction of the preferred cue vector of the cell. The radial axis is in spikes per second, and the angular axis is in degrees. $b$, Distribution of direction differences between preferred eye and preferred cue vectors. The radial axis is number of cells.

We first address the potential ambiguity between eye position during the delay and the first saccade after the delay. On trials with eccentric eye position during the delay, the monkey always made a recentering saccade after the end of the delay. The eye position during the delay was therefore correlated with the direction of this saccade. To resolve this ambiguity, the task was designed so that there were pairs of trials with the same saccade, but different eye positions. For example, in Figure $6 a$, the data points indicated by the small open arrows both correspond to conditions in which the first saccade after the delay was downward. These trials also had the same cue position. Yet activity during eccentric (upward) fixation was $\sim 1.4$ times greater than activity during central fixation (48.4 vs. 35.5 spikes/sec).

To quantify this difference, we measured the effect of eye position for trials with matching saccades by first finding the eccentric eye position that yielded the strongest delay activity averaged over all of the cue locations. We then compared activity for the cue direction that was opposite to this eyeposition in two conditions: first, when the saccade was made from the eccentric eye position and second, when the saccade was made from the center eye position. The same analysis was done for the eccentric eye position with the weakest overall delay activity. Across the population of cells, activity for the best eccentric fixation position averaged 1.51 spikes/sec (SEM, 0.38) greater than activity during central fixation. This difference was statistically significant (paired $t$ test; $p<0.001$ ). Activity for the worst eccentric eye position averaged 1.02 spikes/sec less than activity during central fixation (SEM, 0.20; paired $t$ test, $p<0.0001$ ). The average difference in activity between best and worst eye position was therefore $2.53 \mathrm{spikes} / \mathrm{sec}$. This difference should be comparable with the mean PEV magnitude of 4.02

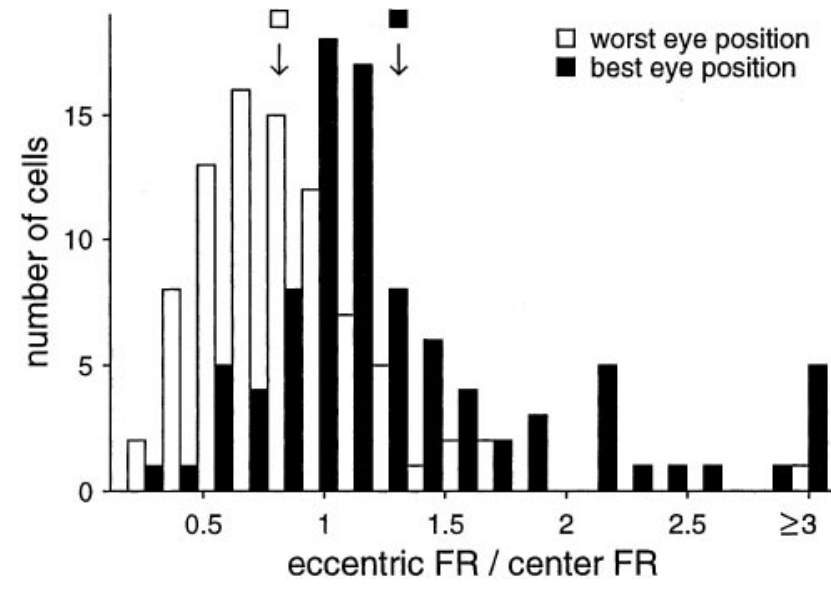

Figure 8. Delay activity for central versus eccentric fixation using saccade-matched trials. Downward arrows indicate the means of the respective distributions. The open bars represent the worst eye position cases, and the filled bars represent the best eye position cases.

spikes/sec, assuming that the PEV reflects eye position and not saccade plan. We conclude that eye position accounts, on average, for $63 \%$ (2.53/4.02) of the PEV.

An eye position index was computed by calculating the firing rate ratio for eccentric and central fixation for trials with matching saccades and cue directions. The distributions of this index for best and worst eye positions are shown in Figure 8. For the best eye position, the mean ratio (eccentric/central) was 1.38 (SEM, 0.09; median, 1.14). For the worst eye position, the mean ratio was 0.82 (SEM, 0.05; median, 0.77). Hence, there was a $20-40 \%$ modulation of firing rate by eye position that was independent of the upcoming saccade.

Next we consider the possibility that delay activity might be modulated not only by the upcoming saccade but also by a superposition of the plans for the next two saccades (i.e., the recentering and choice saccades). In general, it can be shown that a superposition of saccade plans predicts very much the same pattern of activity as an eye position gain field. In Figure 9, we show a hypothetical example based on a simple model in which the activity for eccentric eye positions is a weighted sum of two saccade plans. The plan for the recentering saccade yields the gainfield (i.e., eye position-related) component (Fig. 9, solid symbols), whereas the plan for the choice saccade yields the tuned (i.e., cue position-related) component. This can be expressed mathematically as follows:

$$
F R^{e}(i, j)=w_{1} F R_{i}^{c}+w_{2} F R_{j}^{c} .
$$

In other words, the firing rate during eccentric gaze $\left(F R^{e}\right)$ as a function of eye position ( $i$ ) and cue position $(j)$ is a linear combination of an appropriate pair of firing rates during central fixation $\left(F R^{c}\right)$. The important observation is that the activity for any gaze position can be accounted for given the tuning of the neuron during central fixation. How well does such a model fit the data? We conducted least-squares fits of the model for each cell, allowing $w_{1}$ and $w_{2}$ to vary. We found that the predicted activity accounted for $77 \%$ of the variance of the normalized neuronal activity across the entire population of neurons (Fig. 10). A regression line was fit to the population data in Figure 10, using an algorithm that minimized the least-squared error in both $x$ and $y$. The slope and intercept were 1.1 and -0.03 , respectively.

The model reveals the weight with which each cell represents each saccade plan. These weights can be represented by a scatter 

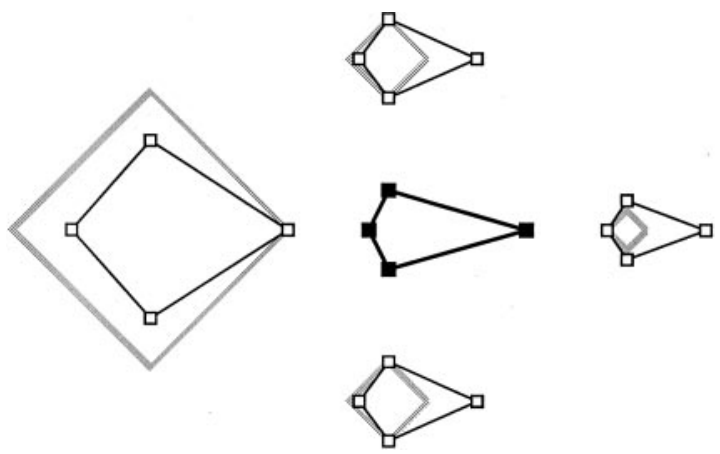

Figure 9. Superposition of saccade plans model. Filled symbols and solid lines in the middle plot represent the spatially tuned response for central fixation. Gray lines in the outer ring of plots represent the gainfield-like component. Open squares with thin lines represent the average of the tuned and gainfield response components.

plot of $w_{1}$ versus $w_{2}$ (Fig. 11). One might expect both plans to be equally represented such that the weights would be clustered along a line of slope 1.0. Another possibility is that individual cells would favor one plan or the other, resulting in two clusters of positive weights along either axis. In fact, the results show that both weights are generally positive, but are negatively correlated $(r=-0.92)$. The more a cell represents one plan, the less it represents the other. Cells with extreme weights for one plan tend to have negative weights for the other, which might indicate some mutual inhibition between the neural representations of the two plans.

\section{Discussion}

Sustained activity consistent with various forms of spatial memory has been observed previously in several regions of the posterior parietal cortex (Snyder, 2000) and prefrontal cortex (Levy and Goldman-Rakic, 2000). Many of these regions also carry eye movement-related activity that could potentially interfere with memory signals. It is therefore important to understand the interaction between memory-related and movement-related activity. In this study, we sought to answer two questions. First, how resistant is memory-related activity to distractions? In this case, we used a motor distractor in the form of a gaze shift that intervenes between the cue and response. A gaze shift would be expected to be accompanied by a host of movement-related signals in the FEF, any of which could be deleterious to the memory signal. The second question concerns how the contents of memory are altered by the gaze shift. In particular, is there a memory of cue location encoded in the delay activity when the gaze is shifted?

We trained monkeys to perform a delayed spatial matching task. During the memory interval of the task, gaze was either fixed in the center of the display or shifted to one of four eccentric locations. We found that the sustained activity during the delay was modulated by gaze shifts in slightly more than one-half the FEF neurons in our sample. In comparison, one-third of the neurons had delay activity that was significantly tuned for the cue location. On average, the magnitudes of the eye and cue position signals were comparable $\left(\sim 0.25\right.$ spikes $\left.\cdot \mathrm{sec}^{-1} \cdot \mathrm{deg}^{-1}\right)$. For this task, the ideal storage mechanism would have been completely insensitive to the change in eye position, because this information was irrelevant for correct performance. However, we found only five neurons $(<5 \%)$ that were significantly tuned for retinal stimulus location and not significantly modulated by gaze shifts.

Using the direction of the population vector as a proxy for the contents of working memory, it was clear that a memory of cue

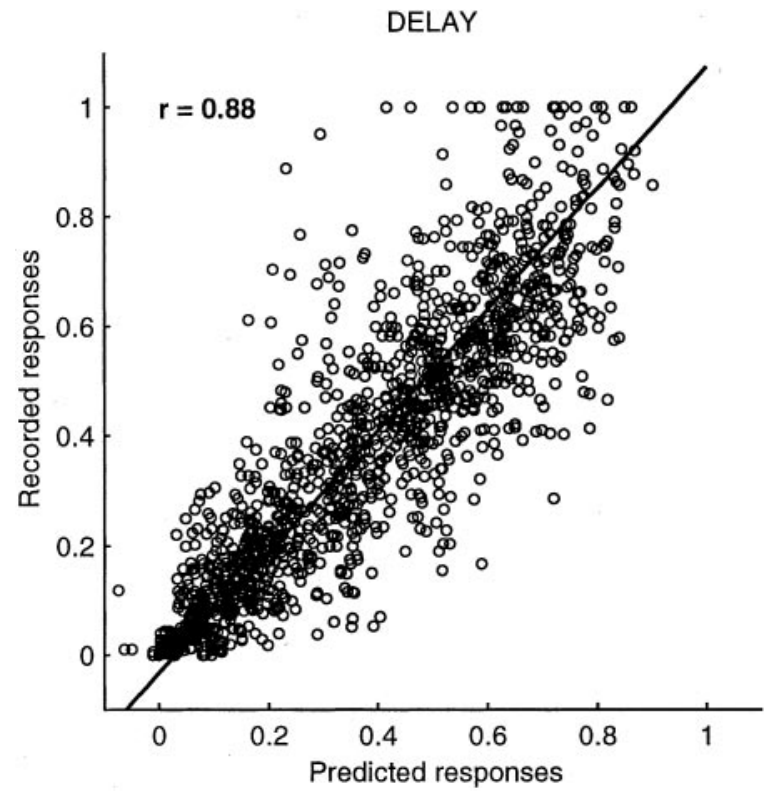

Figure 10. Normalized delay activity and model predictions. Open circles represent the average delay activity sorted by cue direction and eye position compared with the acitvity predicted by the weighted saccade plan model. The set of activities for each cell was normalized to the maximum for that cell. Solid line is the least-mean squares regression (slope $=1.1$; intercept $=-0.03)$. $r$ is the sample correlation coefficient.

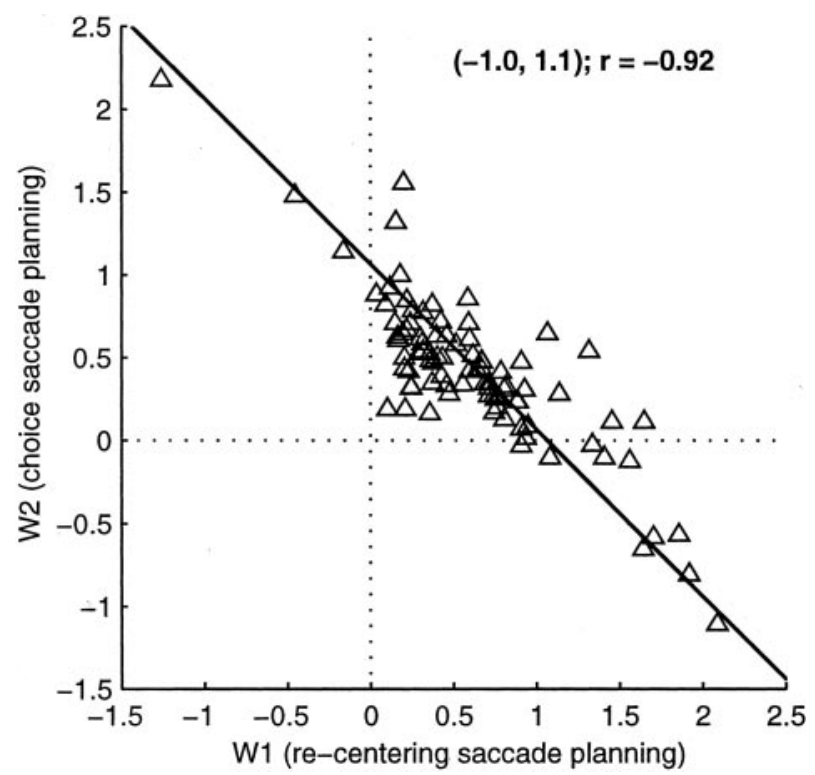

Figure 11. Plot of the weights $(W)$ given to each saccade plan by each neuron. Weights were found by fitting the responses of each neuron with the superposition model. Solid line is leastmean squares regression. Numbers in parentheses are the slope and intercept, respectively. Each open triangle represents the pair of weights for a single neuron. $r$ is the correlation coefficient of the sample.

location relative to the center of the screen was maintained throughout the delay regardless of gaze position. However, the amplitude of the population vector was slightly reduced for eccentric gaze positions. In contrast, at the end of the delay, when the recentering saccade was made, hardly any neurons showed tuned responses, suggesting that the memory trace was temporarily abolished. The tuning reappeared before the final choice saccade. This pattern of results suggests that spatial working memory is buffered outside of the FEF, and that the FEF is in- 
volved in the readout rather than storage of spatial working memory.

There are two general theories regarding gaze-dependent modulation of neuronal activity. The first is the gain field model, which posits that visual responses are modulated by an eye position signal. Eye position gain fields were described in the parietal cortex more than a decade ago (Andersen et al., 1990). Computational theory suggests that the modulation of retinotopic receptive fields by eye position may represent a partial spatial transformation from retinocentric to head-centered coordinates (Zipser and Andersen, 1988). Other work has suggested that the transformation may be completed in premotor cortex (Graziano and Gross, 1998). The second model proposes that visual responses are remapped around the time of saccades via a vector subtraction mechanism (Goldberg and Bruce, 1990; Umeno and Goldberg, 1997, 2001; Quaia et al., 1998). Goldberg and colleagues proposed vector subtraction as a mechanism for updating saccade plans based on the recent history of eye movements and rejected the idea that it might contribute to an explicit representation of target location in head-centered coordinates.

The gain field model is the more general of the two, in that it allows the eye position signal to be separable from the visual response. This model can accommodate gaze-dependent modulation in the form of a planar gain field (Andersen et al., 1990) (Fig. 6a), as well as cases in which eccentric eye position uniformly enhances or diminishes delay activity, consistent with U-shaped or inverted-U-shaped gain fields $(b)$. We should emphasize that examples of the latter were quite rare. The gain field model also allows for the possibility that a neuron could be modulated by eye position even when its visual response is weak or untuned. This could account for our observation that 22 of 91 (24\%) neurons showed significant tuning for eye position but not for cue location.

Remapping via vector subtraction makes the more specific prediction that activity should increase when gaze is shifted away from the RF and decrease when gaze is shifted toward the RF. In this regard, our data clearly support the remapping hypothesis. Furthermore, we found that a simple model of a superposition of saccade plans accounted for $\sim 80 \%$ of the variance in firing rate across the population of FEF neurons. This model showed a clear trade-off between the plans for the recentering and choice saccades. The superposition model accounts for gain field-like responses without any need for an explicit eye position signal. Rather, the model entails a queuing of saccade plans in a manner that is consistent with the remapping hypothesis.

The superposition model may also account for a curious feature of our data. We observed that the memory trace was preserved after gaze shifted to an eccentric position but was abolished after the recentering saccade. In both cases, the monkey was simply following the movement of the fixation target, and thus it is not clear why the two saccades should affect the memory signal differently. One possibility is that the memory trace simply becomes more fragile with the passage of time and therefore more susceptible to perturbations. A more interesting possibility is that, when the monkey makes an eccentric gaze shift, it is adding a saccade plan (for the recentering saccade) to the already existing plan for the choice saccade. The addition of the second plan does not disrupt the storage of the first. However, when the monkey executes the recentering saccade, rather than simply subtracting that plan from the memory trace, there is a general reset signal that temporarily purges both plans from the FEF. The plan for the choice saccade is then restored from a memory buffer outside of the FEF.
To summarize, the results of this study support a view of the FEF as an area that reads out information from memory buffers for planning saccades. The FEF may be involved in executive processes that operate on the contents of working memory and thereby complement areas involved in working memory storage (Smith and Jonides, 1999). These results lay the groundwork for additional studies using multisite recordings to elucidate the interactions between regions that perform memory storage and retrieval, respectively. FEF activity may represent multiple saccade plans by a superposition mechanism. Adding a second plan does not seem to interfere substantially with a previously existing plan. However, executing the second plan results in the dumping of both plans and the subsequent reloading of the original plan. Additional work will be needed to determine how many saccade plans can coexist in the FEF and if the resetting mechanism depends on the order in which the plans are loaded and executed. The current observations neither require nor rule out the possibility of a patent eye position signal, but the demonstration of such a signal in FEF will require additional work.

\section{References}

Andersen RA, Mountcastle VB (1983) The influence of the angle of gaze upon the excitability of light-sensitive neurons of the posterior parietal cortex. J Neurosci 3:532-548.

Andersen RA, Essick GK, Siegel RM (1985) Encoding of spatial location by posterior parietal neurons. Science 230:450-458.

Andersen RA, Bracewell RM, Barash S, Gnadt JW, Fogassi L (1990) Eye position effects on visual, memory, and saccade-related activity in areas LIP and 7a of macaque. J Neurosci 10:1176-1198.

Barbas H, Mesulam MM (1981) Organization of afferent input to subdivisions of area 8 in the rhesus monkey. J Comp Neurol 200:407-431.

Bremmer F, Pouget A, Hoffman K-P (1998) Eye position encoding in the macaque posterior parietal cortex. Eur J Neurosci 10:153-160.

Bruce CJ, Goldberg ME (1985) Primate frontal eye field. I. Single neurons discharging before saccades. J Neurophysiol 53:603-635.

Bruce CJ, Goldberg ME, Bushnell MC, Stanton GB (1985) Primate frontal eye field. II. Physiological and anatomical correlates of electrically evoked eye movements. J Neurophysiol 54:714-734.

Ferraina S, Pare M, Wurtz RH (2000) Disparity sensitivity of frontal eye field neurons. J Neurophysiol 83:625-629.

Fujii N, Mushiake H, Tanji J (1998) Intracortical microstimulation of bilateral frontal eye field. J Neurophysiol 79:2240-2244.

Galletti C, Battaglini PP, Fattori P (1995) Eye position influence on the parieto-occipital area PO (V6) of the macaque monkey. Eur J Neurosci 9:410-413.

Goldberg ME, Bruce CJ (1990) Primate frontal eye fields. III. Maintenance of a spatially accurate saccade signal. J Neurophysiol 64:489-508.

Graziano MSA, Gross CG (1998) Visual responses with and without fixation: neurons in premotor cortex encode spatial location independently of eye position. Exp Brain Res 118:373-380.

Graziano MSA, Hu TX, Gross CG (1997) Visuospatial properties of ventral premotor cortex. J Neurophysiol 77:2268-2292.

Judge SJ, Richmond BJ, Chu FC (1980) Implantation of magnetic search coils for measurement of eye position: an improved method. Vision Res 20:535-538.

Levy R, Goldman-Rakic PS (2000) Segregation of working memory functions within the dorsolateral prefrontal cortex. Exp Brain Res 133:23-32.

Mohler CW, Goldberg ME, Wurtz RH (1973) Visual receptive fields of frontal eye field neurons. Brain Res 61:385-389.

Mushiake H, Tanatsugu Y, Tanji J (1997) Neuronal activity in the ventral part of premotor cortex during target-reach movement is modulated by direction of gaze. J Neurophysiol 78:567-571.

Mushiake H, Fujii N, Tanji J (1999) Microstimulation of the lateral wall of the intraparietal sulcus compared with the frontal eye field during oculomotor tasks. J Neurophysiol 81:1443-1448.

Opris I, Barborica A, Ferrera VP (2001) On the gap effect for saccades evoked by electrical microstimulation of frontal eye fields in monkeys. Exp Brain Res 138:1-7.

Quaia C, Optican LM, Goldberg ME (1998) The maintenance of spatial 
accuracy by the perisaccadic remapping of visual receptive fields. Neural Netw 11:1229-1240.

Robinson DA, Fuchs AF (1969) Eye movements evoked by stimulation of frontal eye fields. J Neurophysiol 32:637-648.

Russo GS, Bruce CJ (1993) Effect of eye position within the orbit on electrically elicited saccadic eye movements: a comparison of the macaque monkey's frontal and supplementary eye fields. J Neurophysiol 69:800-818.

Salinas E, Thier P (2000) Gain modulation: a major computational principle of the central nervous system. Neuron 27:15-21.

Schall JD, Morel A, King DJ, Bullier J (1995a) Topography of visual cortex connections with frontal eye field in macaque: convergence and segregation of processing streams. J Neurosci 15:4464-4487.

Schall JD, Hanes DP, Thompson KG, King DJ (1995b) Saccade target selection in frontal eye field of macaque. I. Visual and premovement activation. J Neurosci 15:6905-6918.

Schlag J, Schlag-Rey M, Pigarev I (1992) Supplementary eye field: influence of eye position on neural signals of fixation. Exp Brain Res 90:302-306.
Siegel RM (1998) Representation of visual space in area 7a neurons using the center of mass equation. J Comput Neurosci 5:365-381.

Smith EE, Jonides J (1999) Storage and executive processes in the frontal lobes. Science 283:1657-1661.

Snyder LH (2000) Coordinate transformations for eye and arm movements in the brain. Curr Opin Neurobiol 10:747-754.

Tian J, Schlag J, Schlag-Rey M (2000) Testing quasi-visual neurons in the monkey's frontal eye field with the triple-step paradigm. Exp Brain Res 130:433-440.

Umeno MM, Goldberg ME (1997) Spatial processing in the monkey frontal eye fields. I. Predictive visual responses. J Neurophysiol 73:1988-2003.

Umeno MM, Goldberg ME (2001) Spatial processing in the monkey frontal eye fields. II. Memory responses. J Neurophysiol 86:2344-2352.

Zar JH (1999) Biostatistical analysis. Upper Saddle River, NJ: Prentice Hall. Zipser D, Andersen RA (1988) A back-propagation programmed network that simulates response properties of a subset of posterior parietal neurons. Nature 331:679-684. 